\title{
PVDF-SIC Composite Thick Films an Effective ESD Composition for Growing Anti-Static Applications
}

\author{
Nina Joseph ${ }^{\mathrm{a}, \mathrm{b}}$, Jobin Varghese ${ }^{\mathrm{a}, \mathrm{b}}$, Mailadil Thomas Sebastian ${ }^{\mathrm{a}, \mathrm{b}^{*}}$ \\ ${ }^{a}$ Microelectronics Research Unit, Faculty of Information Technology and Electrical Engineering, University of \\ Oulu, Finland, P.O. Box 4500, FI-90014. \\ ${ }^{\mathrm{b}}$ Functional Materials Group, Materials Science \& Technology Division, CSIR NIIST, Thiruvananthapuram, \\ Kerala, India 695019 \\ *E-mail: mailadils@yahoo.com
}

\begin{abstract}
Thin polyvinylidene fluoride - silicon carbide (PVDF-SiC) shielding composite films were prepared by a simple solution casting process. The X-ray diffraction (XRD) and scanning electron microscope (SEM) analysis show the presence of both polymer and filler with no reaction between them. The coefficient of thermal expansion (CTE) and tensile strength of the composite exhibit a decreasing tendency, while that of conductivity and electromagnetic interference (EMI) shielding values increase with filler loading. The $\varepsilon_{\mathrm{r}}$, and $\tan \delta$ of the composites were also found to increase with filler loading in the frequency range of $8.2-18 \mathrm{GHz}$, which also influence the shielding behaviour of the composite. The high filler (60 vol\% of $\mathrm{SiC}$ ) loaded composite of $1 \mathrm{~mm}$ thickness exhibits direct current (DC) conductivity of the order of $10^{-5} \mathrm{~S} / \mathrm{m}$ indicating its potential for electrostatic discharge shielding applications. This is further confirmed from its EMI SE value of $12 \mathrm{~dB}$, which corresponds $94 \%$ attenuation. The composite exhibits a partially reflecting and absorbing shielding nature, without any dominant shielding mechanism. The alternating current (AC) conductivity and skin depth value of the composites exhibit an inverse relationship with each other and influence the shielding thickness. The attenuating power of the PVDF-SiC composite with 60 volume \% (vol\%) silicon carbide (SiC) changes from $84 \%$ to $99 \%$, with an increase in sample thickness from 0.5 to $2 \mathrm{~mm}$. Hence, thin and flexible paper-like EMI shield of PVDF-SiC composite can be easily prepared by simple, cost-effective approach for various shielding applications like electrostatic discharge shielding as well as EMI shielding.
\end{abstract}

Keywords: PVDF, Silicon carbide, composite, EMI shielding, films

\section{Introduction}

Electrostatic discharge (ESD) or anti-static materials play the role of obstructing undesirable electromagnetic wave radiations and hence gaining demand and interest in this growing technology [1-5]. The explosive growth in the 
electronic and communication industry also creates interference among the electronic systems, which can cause a disturbance, poor device operation, as well as leakage of information etc. Electrostatic discharge shielding materials can protect the sensitive and sophisticated devices, components and circuits from electrostatic discharge. The ESD protection is the most common issues in the modern electronics and technology, its application ranges ESD-work place products, shoes (eg. EN 61340-5-1> $100 \mathrm{k}$ ohms and < 35 M ohms), clothing, floor covering, transport system, measuring instruments, packaging, cleaning [6]. These materials have conductivity between insulating and conducting materials [7-10]. Light, thin and cost-effective shielding materials with ease of preparation and exhibiting good shielding efficiency and physical properties are important and in great demand. Such materials can provide the flexibility to apply for various devices as well surfaces. Polymer composite approach can deliver lightweight composites with low cost integration of the physical properties of the polymer to that of the electrical and thermal properties of the filler [4]. Materials like metals and carbon materials are extensively studied for shielding applications and have good shielding properties due to their good electrical properties. However, preparation problems due to high density of metals as well as dispersion difficulties of carbon materials make the development of thin films challenging $[3,4]$.

$\mathrm{SiC}$ has been used as a promising material for wide range of applications such as thermal insulation, high frequency electron, antiradiation because of its good physical properties such as high hardness and thermal properties like high thermal stability, low coefficient of thermal expansion. Due to its good physical and chemical properties, SiC can offer a promising lightweight and stable shielding material [11-17]. The few reports on shielding materials based on $\mathrm{SiC}$ are mostly multi-composite, with complex preparation technique due to the relatively low shielding efficiency of $\mathrm{SiC}$ [11-17]. However, the shielding efficiency of $\mathrm{SiC}$ is enough for low shielding applications like electrostatic discharge shielding. The present work is an attempt to effectively utilize the good properties of $\mathrm{SiC}$ by developing a thin flexible paper like films through polymer composite approach. PVDF is chosen as the base polymer due to its good physical and chemical properties [18-20]. The present work involves the preparation of PVDF-SiC composite films and investigating its thermal, mechanical, dielectric and shielding properties as well as the influence of conductivity and shielding thickness as an effort to improve the shielding efficiency of the composite.

\section{Methodology and characterization}


The PVDF-silicon carbide (PVDF-SiC) composite films were prepared by solution casting method. The PVDF polymer was obtained from Nikunj Industries, Mumbai, India, while that of the silicon carbide (SiC) powder was procured from Carborundum Universal Ltd, Mumbai, India.

The SiC powder dispersed in dimethylformamide (DMF) was added to the dissolved PVDF solution in the required vol\%, followed by ultrasonicate mixing. The obtained mixture was poured to a heated glass plate at about $50{ }^{\circ} \mathrm{C}$ and thin composite paper films were obtained as shown in figure 1 by drying. The dried films were hot-pressed to various thickness and shapes according to the requirements for various measurements. A maximum filler loading of 60 vol\% was achieved.

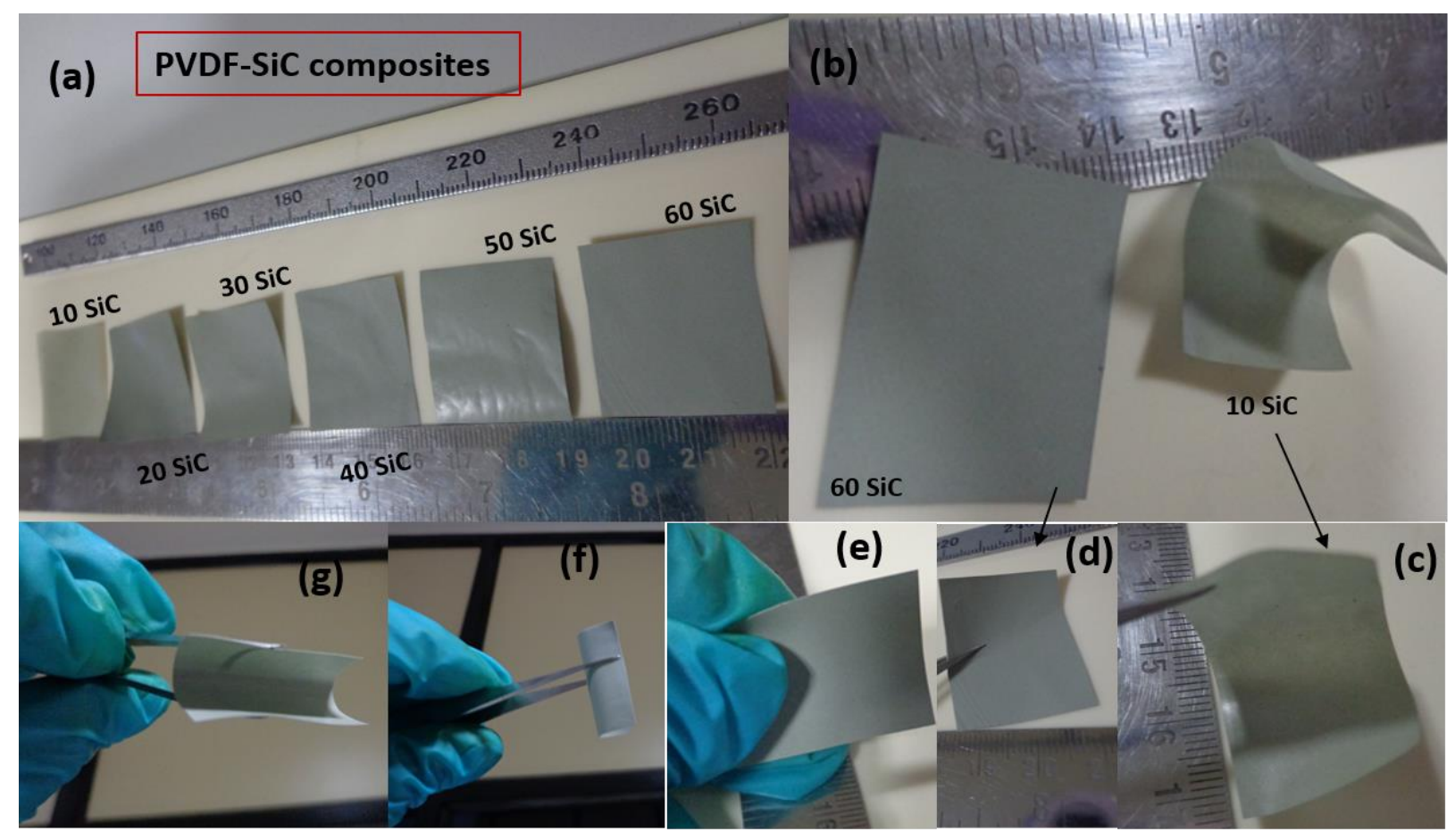

Figure 1: Photographic images of PVDF-SiC composite paper films (a) SiC loading from 10-60 vol\% (b) SiC loading of 10 and $60 \mathrm{vol} \%$ (c-g) indicating its flexibility.

Cylindrical samples of $10 \times 10 \mathrm{~mm}$ dimension was required for coefficient of thermal expansion (CTE) measurement and for tensile measurement, dumb-bell shaped samples of $4 \mathrm{~mm}$ width and 1.5-2 $\mathrm{mm}$ thickness was used. EMI shielding measurements were performed using rectangular samples with dimensions $22.86 \times 10.80 \times 1.00 \mathrm{~mm}$ (X band) and $15.80 \times 7.90 \times 1.00 \mathrm{~mm}$ (Ku band). The rectangle samples of same dimensions were used for dielectric measurements over these frequency ranges and for conductivity measurements. Thickness effects were studied by varying the sample thickness. The XRD of the polymer, filler and the composite were obtained using $\mathrm{CuK} \alpha$ radiation 
(X'Pert PRO MPD X-ray diffractometer, PANalytical, Almelo, Netherlands) and the microstructure of the composite by SEM (JEOL, JSM-5600LV, Tokyo, Japan). CTE was measured by thermos mechanical analyzer (SII Nanotechnology Inc., Tokyo, Japan) while tensile property measurement by Universal Testing Machine (Hounsfield, H5K-S UTM, Redhill, UK) at a crosshead speed of $50.0 \mathrm{~mm} \mathrm{~min}^{-1}$. DC conductivity of the samples was measured by Four probe method using current source Aplab $9710 \mathrm{P}$ and nano voltmeter Keithley $2182 \mathrm{~A}$. The complex permittivity was obtained with an accuracy of about $2 \%$. using Agilent software module 85071E from the measured scattering parameters using vector network analyzer (Agilent Technologies E5071C, ENA series, $300 \mathrm{kHz}-20 \mathrm{GHz}, \mathrm{CA}$ ) by the waveguide technique. The EMI shielding effectiveness were obtained from the scattering parameters using X and $\mathrm{Ku}$ band wave guides measured by vector network analyzer.

\section{Results and discussions}

\subsection{Structure and microstructure}

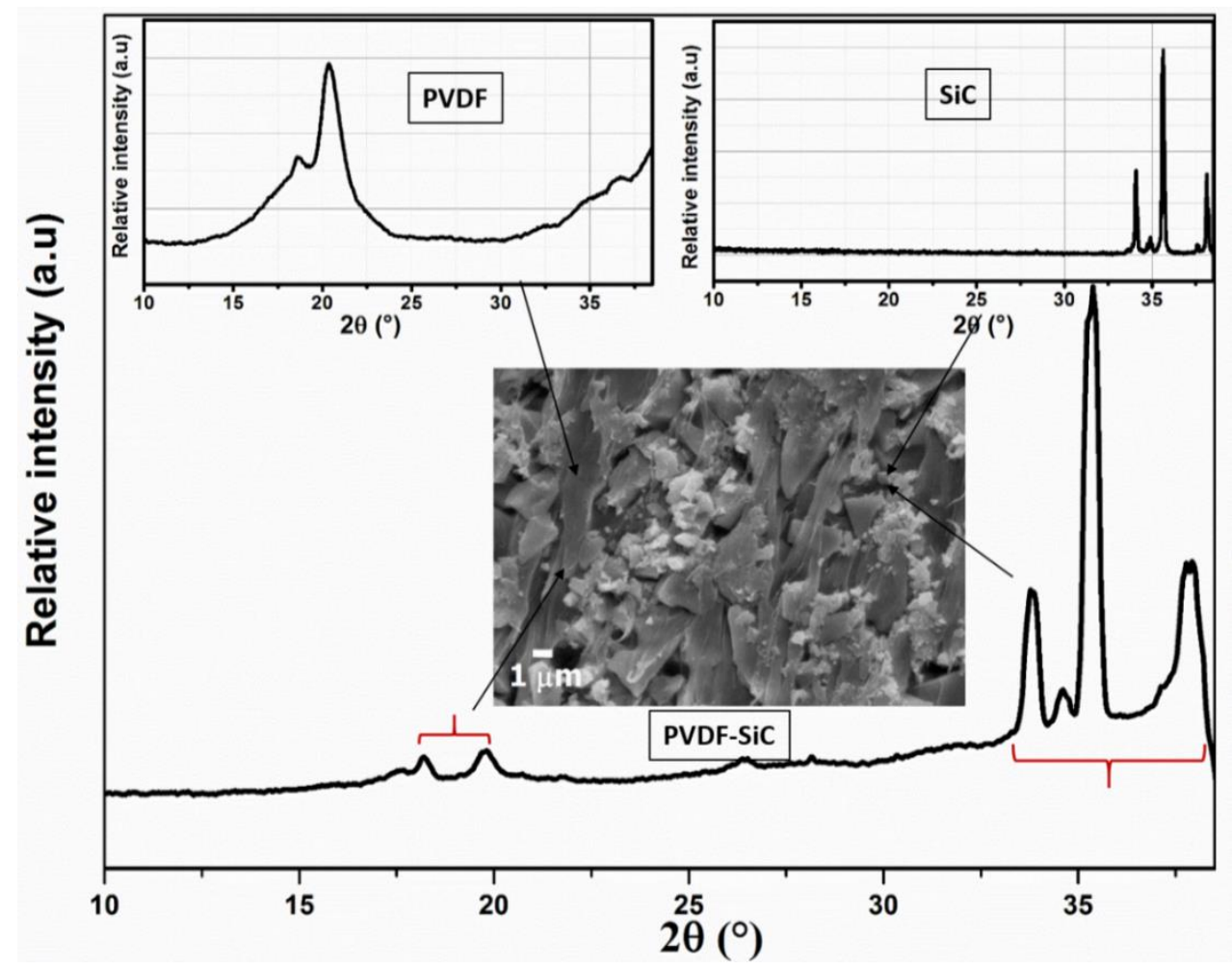

Figure 2: XRD pattern of the PVDF -SiC composite with 60 vol\% filler loading (Inset figures show the SEM fractogram image of the same composite, XRD pattern of pure PVDF and SiC ceramic).

The XRD pattern of PVDF-SiC composite with 60 vol\% of SiC loading shown in figure 2 indicates the presence of both PVDF and SiC and in agreement with the XRD patterns of PVDF and SiC given in the inset of figure 2 [19]. The 
diffraction peaks at $2 \theta$ values of $34.1^{\circ}, 35.6^{\circ}$ and $38^{\circ}$ in the XRD pattern of the PVDF-SiC corresponds to the hexagonal SiC (JCPDS No. 89-2223). The XRD pattern indicates the absence of any reaction between the polymer and the $\mathrm{SiC}$, and is further clear from the SEM image of the fractogram of the same composite. The microstructure of the composite also indicates that the SiC particles are well distributed in the PVDF matrix, which is important for good physical and chemical properties.

\subsection{Thermal and mechanical properties}

The coefficient of thermal expansion and tensile strength of the composites shown in figure 3 exhibited a decreasing tendency with increase in filler loading. This is expected due to the decrease in the loosely bounded polymer chains, poor filler matrix interface, and increase in porosity with filler loading [21-23]. The thermal expansion coefficient decreased to 120 and $35 \mathrm{ppm} /{ }^{\circ} \mathrm{C}$ respectively by the addition of 10 and $60 \mathrm{vol} \%$ of $\mathrm{SiC}$ into the PVDF. Similarly, for the same increase in filler loading, the tensile strength decreased from 29 to $7 \mathrm{MPa}$ with decrease in elongation from

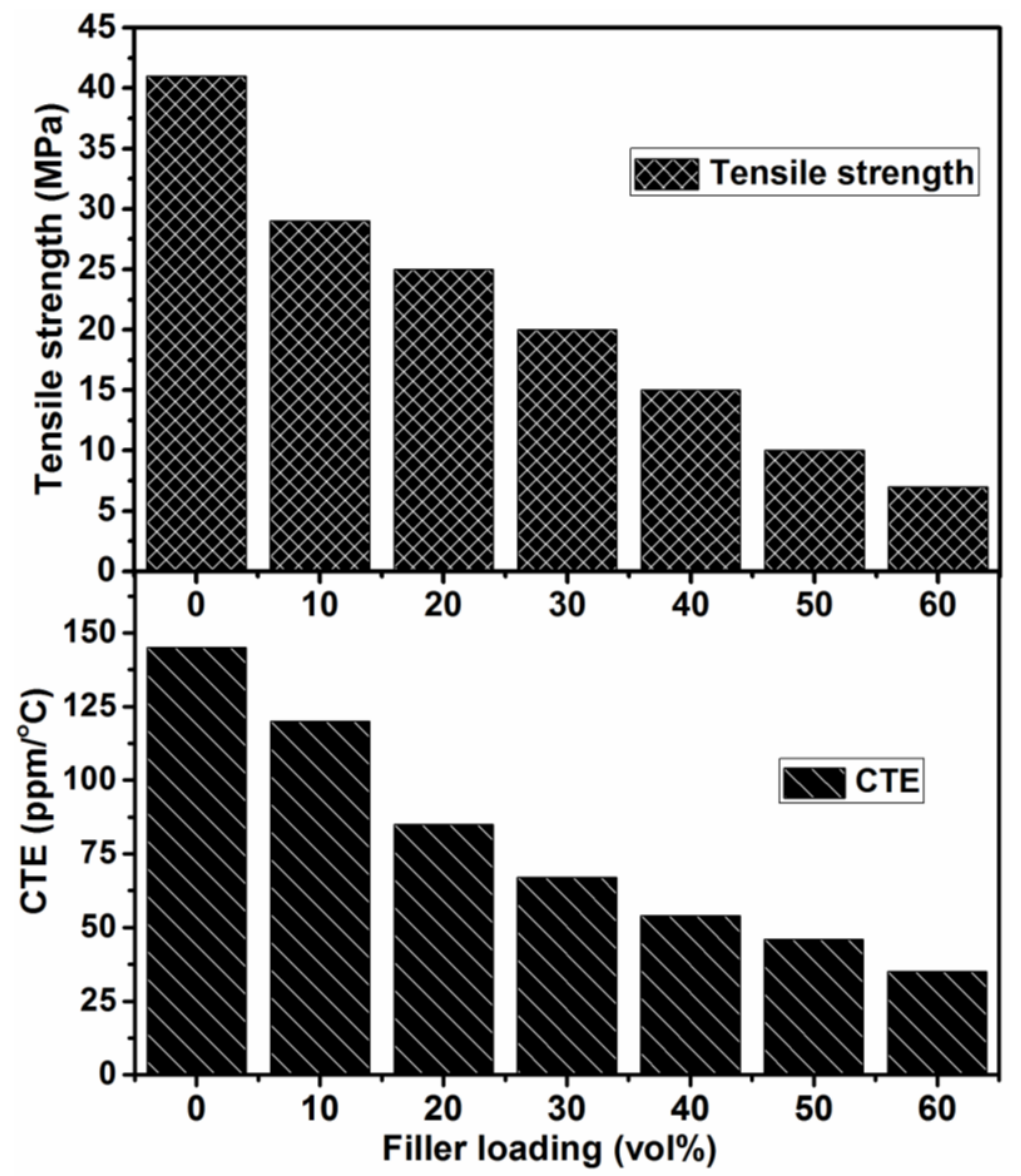

Figure 3: CTE and tensile strength of PVDF-SiC composite with different filler loading. 
8.6 to $3 \%$. It is clear from the figure 1 that this composite maintains its flexibility even at highest filler loading of 60 vol\%.

\subsection{Dielectric properties}

The influence of filler loading and frequency on the dielectric properties of PVDF-SiC composite is shown in figure 4. Both relative permittivity $\left(\varepsilon_{\mathrm{r}}\right)$ and tan $\delta$ increase with increase in filler loading indicating that the major contribution to the dielectric properties is from the filler SiC. Dielectric properties display almost stable over this frequency range. The $\varepsilon_{\mathrm{r}}$ and shows a slight decreasing trend while $\tan \delta$ and a slightly increasing tendency with increase in frequency [24]. The addition of $60 \mathrm{vol} \% \mathrm{SiC}$ to PVDF with a $\varepsilon_{\mathrm{r}}=2.4$ and a $\tan \delta 0.04$ results in the increase of $\varepsilon_{\mathrm{r}}$ and $\tan \delta$ to 19.8 and 0.21 respectively. This is attributed to the larger $\varepsilon_{\mathrm{r}}$ and $\tan \delta$ values of the $\mathrm{SiC}$ ceramic [25], which in turn influence the conductivity and skin depth of the composite.

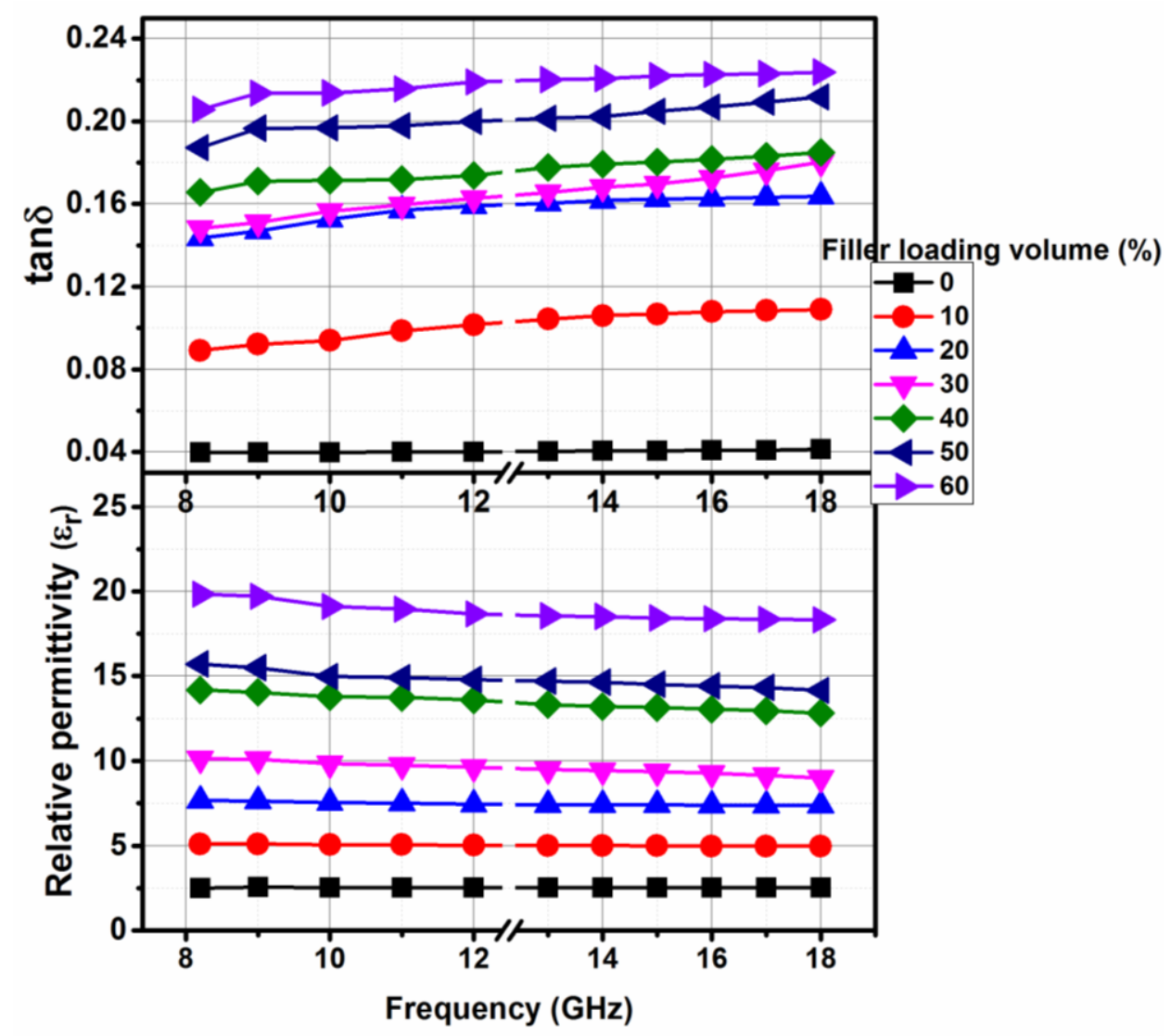

Figure 4: Dielectric properties of PVDF-SiC composite with different filler loading in the frequency range of 8.2-18 GHz. 


\subsection{Conductivity and Skin depth}

The conductivity (AC and DC) and skin depth shown in the figure 5 represent the relationship between conductivity and skin depth as well as their dependence on frequency and filler loading. Addition of $60 \mathrm{vol} \%$ of SiC results in the increase of DC conductivity (inset of figure 5) by $10^{9}$ times from $10^{-14} \mathrm{~S} / \mathrm{m}$ (pure PVDF) to that of $7 \times 10^{-5} \mathrm{~S} / \mathrm{m}$ for PVDF-60 SiC composite. The conductivity values of these composites make it suitable for electrostatic discharge shielding applications [10].

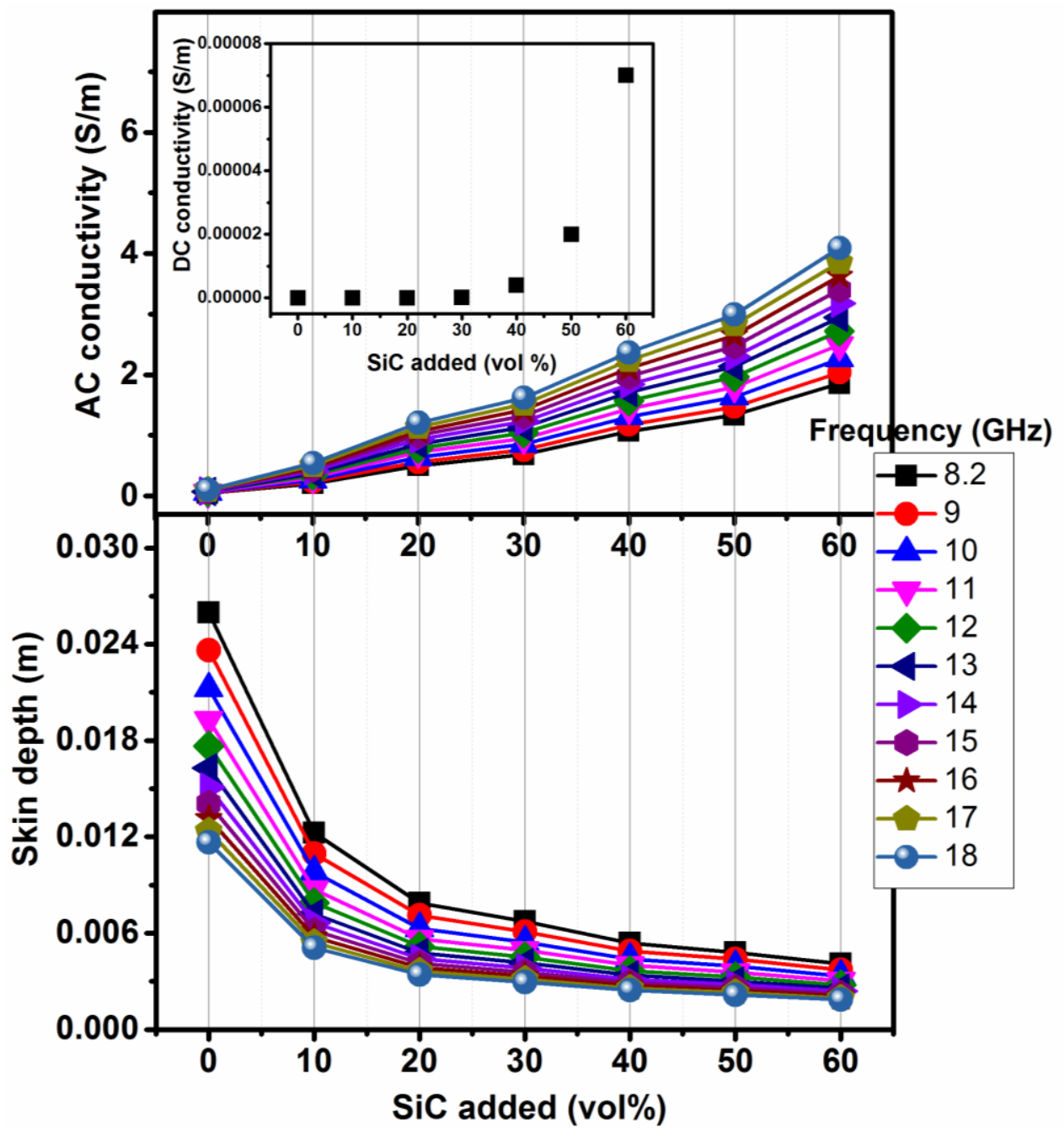

Figure 5: AC conductivity (Inset figure DC conductivity) and skin depth of the PVDF-SiC composite with filler loading in the frequency range of $8-18 \mathrm{GHz}$

Similar to DC conductivity, AC conductivity also shows increasing trend with filler loading and is calculated as $2 \pi \mathrm{f} \varepsilon_{0} \varepsilon$ ", where $\mathrm{f}$ is the frequency, $\varepsilon_{0}$ is the relative permittivity of the free space and $\varepsilon$ " is the imaginary part of the 
permittivity [18]. AC conductivity depends on the frequency, which is in agreement with the results obtained as it shows increasing nature with frequency. PVDF-60 SiC composite exhibits change in AC conductivity from 1.8 to 4.1 $\mathrm{S} / \mathrm{m}$ in the frequency range of $8.2-18 \mathrm{GHz}$. At high frequency of $18 \mathrm{GHz}, 60 \mathrm{vol} \% \mathrm{SiC}$ addition to pure PVDF results in increase of AC conductivity by 4 times. This improvement in the conductivity may corresponds to the good dielectric properties of the ceramic filer compared to the polymer matrix as well as to good dispersion.

Skin depth determines the effective depth of electric field attenuation of a material. It is given as $(\pi f \mu \sigma)^{-1 / 2}$, where $\mathrm{f}$ is the frequency, $\mu$ is the magnetic permeability $=\mu_{0} \mu_{\mathrm{r}}, \mu_{\mathrm{r}}$ is the relative permeability, $\mu_{0}=10=4 \times 10^{-7} \mathrm{H} / \mathrm{m}$ and $\sigma$ is the conductivity in $\mathrm{S} / \mathrm{m}[19]$. Hence, it gives information of the distance at which the value of the field drops to 1/e of the incident value and is inversely proportional to frequency and conductivity. The variation of skin depth of PVDF$\mathrm{SiC}$ composite with frequency and filler loading shown in figure 5 is in agreement with the above-mentioned expected results. The skin depth decreases by half in the measured frequency range of 8.2 to $18 \mathrm{GHz}$ for PVDF-60 SiC composite due to the increase in conductivity with frequency. The addition of $60 \mathrm{vol} \% \mathrm{SiC}$ leads to a decreases of skin depth of the PVDF polymer by ten times from $0.01 \mathrm{~m}$ to $0.002 \mathrm{~m}$. Hence, the skin depth gives the sample thickness for effective attenuation and is clear that for highly conducting samples and at high frequency, skin depth will be small.

\subsection{EMI shielding properties}

The ability of a material to shield the electromagnetic radiation is given by the EMI shielding effectiveness (EMI SE) and it consists of shielding by absorption $\left(\mathrm{SE}_{\mathrm{A}}\right)$ and reflection $\left(\mathrm{SE}_{\mathrm{R}}\right)$. It is given as $\mathrm{SE}_{\mathrm{A}}=-10 \log \left[(\mathrm{S} 21)^{2} /\left(1-\mathrm{S} 11^{2}\right)\right.$ and $\mathrm{SE}_{\mathrm{R}}=-10 \log \left(1-\mathrm{S} 11^{2}\right)$, where $\mathrm{S} 11$ and $\mathrm{S} 12$ are the magnitude of the scattering parameters [26-28]. Figure 6 shows the filler loading and frequency dependence of the EMI SE, $\mathrm{SE}_{\mathrm{A}}$ and $\mathrm{SE}_{\mathrm{R}}$ of PVDF-SiC composites of $1 \mathrm{~mm}$ thickness measured by varying the filler loading at frequencies 8.2, 10, 12, 15 and $18 \mathrm{GHz}$. The EMI SE of PVDF is very low $(0.4 \mathrm{~dB})$ owing to its low dielectric and conducting properties. However, the addition of $\mathrm{SiC}$ filler causes an observable enhancement in its EMI SE value as clear from the figure 6. The total EMI SE increases to $12 \mathrm{~dB}$ over the measured frequencies by the addition of $60 \mathrm{vol} \%$ of SiC, The sensitivity of the EMI SE with filler loading may be due to the increased presence of filler that creates more connecting paths, which eventually leads to the improvement in the shielding properties. The PVDF-60 SiC composite exhibits an attenuation of $94 \%$, which is suitable for low shielding applications like electrostatic discharge shielding. The contribution from $\mathrm{SE}_{\mathrm{A}}$ and $\mathrm{SE}_{\mathrm{R}}$ to the $\mathrm{EMI} \mathrm{SE}$ is found to be 
almost equal, making this composite a partially reflecting and absorbing shielding material. The contribution from $\mathrm{SE}_{\mathrm{A}}$ and $\mathrm{SE}_{\mathrm{R}}$ respectively is around 5 and $6 \mathrm{~dB}$ in the measured frequencies for highest filler loaded PVDF-SiC composite. This corresponds to the partial conducting nature of the samples owing to the low conductivity as well as low dielectric loss of the $\mathrm{SiC}$ ceramic compared to highly conducting fillers [20] and to the small sample thickness as discussed earlier. Similarly, to the EMI SE, both $\mathrm{SE}_{\mathrm{A}}$ and $\mathrm{SE}_{\mathrm{R}}$ increases with filler loading.

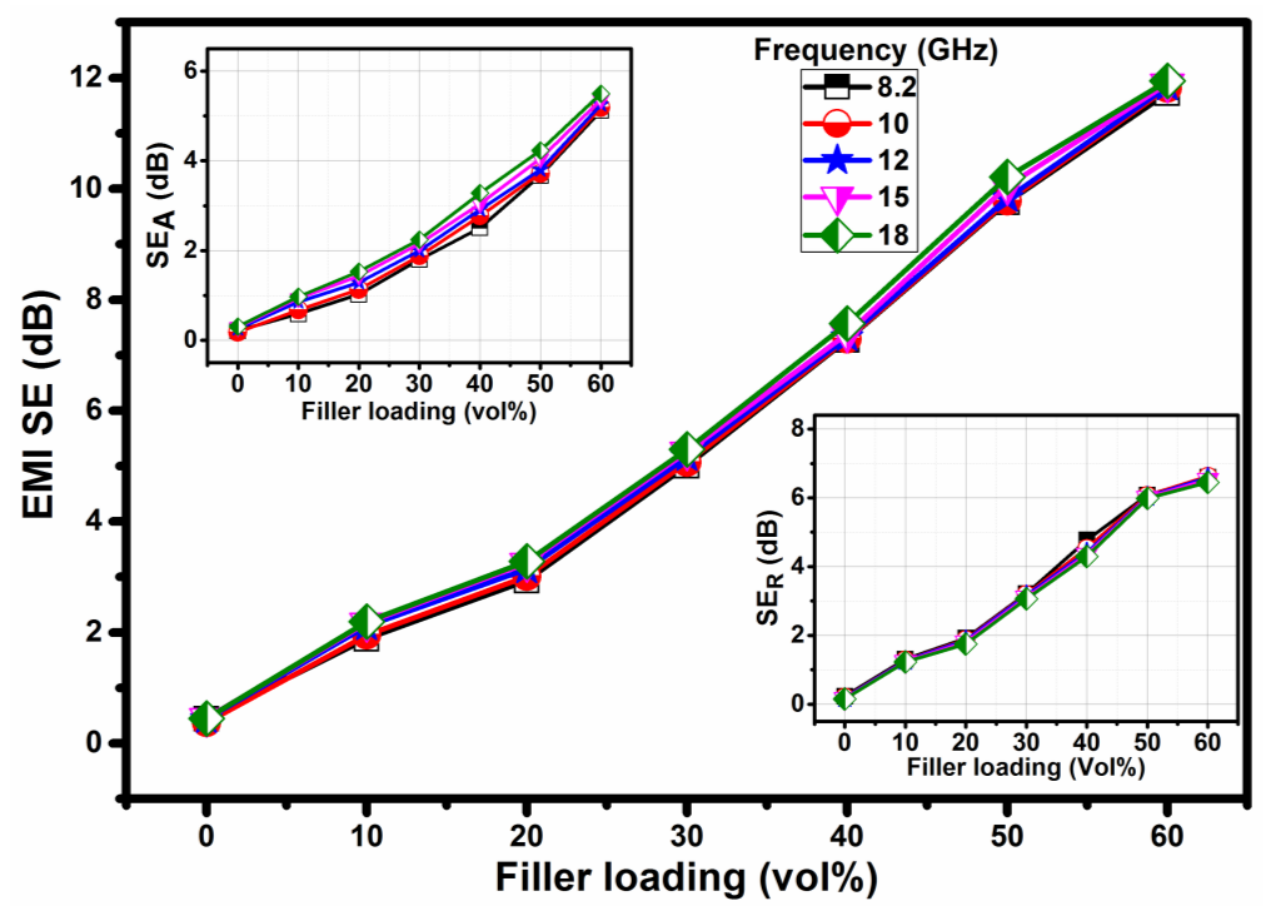

Figure 6: $\mathrm{EMI} \mathrm{SE}$ (Inset figures: $\mathrm{SE}_{\mathrm{A}}$ and $\mathrm{SE}_{\mathrm{R}}$ ) of the PVDF-SiC composite with filler loading at frequencies $8.2,10,12,15$ and $18 \mathrm{GHz}$.

The shielding thickness is an important parameter to get the required shielding, which depends on the shielding material conductivity and frequency of measurement. Figure 7 gives the dependence of EMI SE, $\mathrm{SE}_{\mathrm{A}}$ and $\mathrm{SE}_{\mathrm{R}}$ of PVDF-60 SiC composite at the mid frequencies of the $\mathrm{X}$ and $\mathrm{Ku}$ band of 10 and $15 \mathrm{GHz}$. The shielding properties is found to improve with thickness as the EMI SE increase from 8 to $20 \mathrm{~dB}$ with increase of thickness from 0.5 to $2 \mathrm{~mm}$. Similarly, the $\mathrm{SE}_{\mathrm{A}}$ changes from 3.5 to $9.5 \mathrm{~dB}$, while that of $\mathrm{SE}_{\mathrm{R}}$ from 4.5 to $10 \mathrm{~dB}$ for the same change in sample thickness at the measured frequencies. Hence, this composite can be tuned suitable for wide range of shielding applications from low shielding applications of electrostatic discharge shielding to EMI shielding applications by changing the sample thickness by $1.5 \mathrm{~mm}$. The ability of the composite to attenuate the electromagnetic radiation 
increases from $84 \%$ to $99 \%$ with increase in sample thickness. This result correlates to the skin depth of the composite, which is around $2 \mathrm{~mm}$ (figure 5).

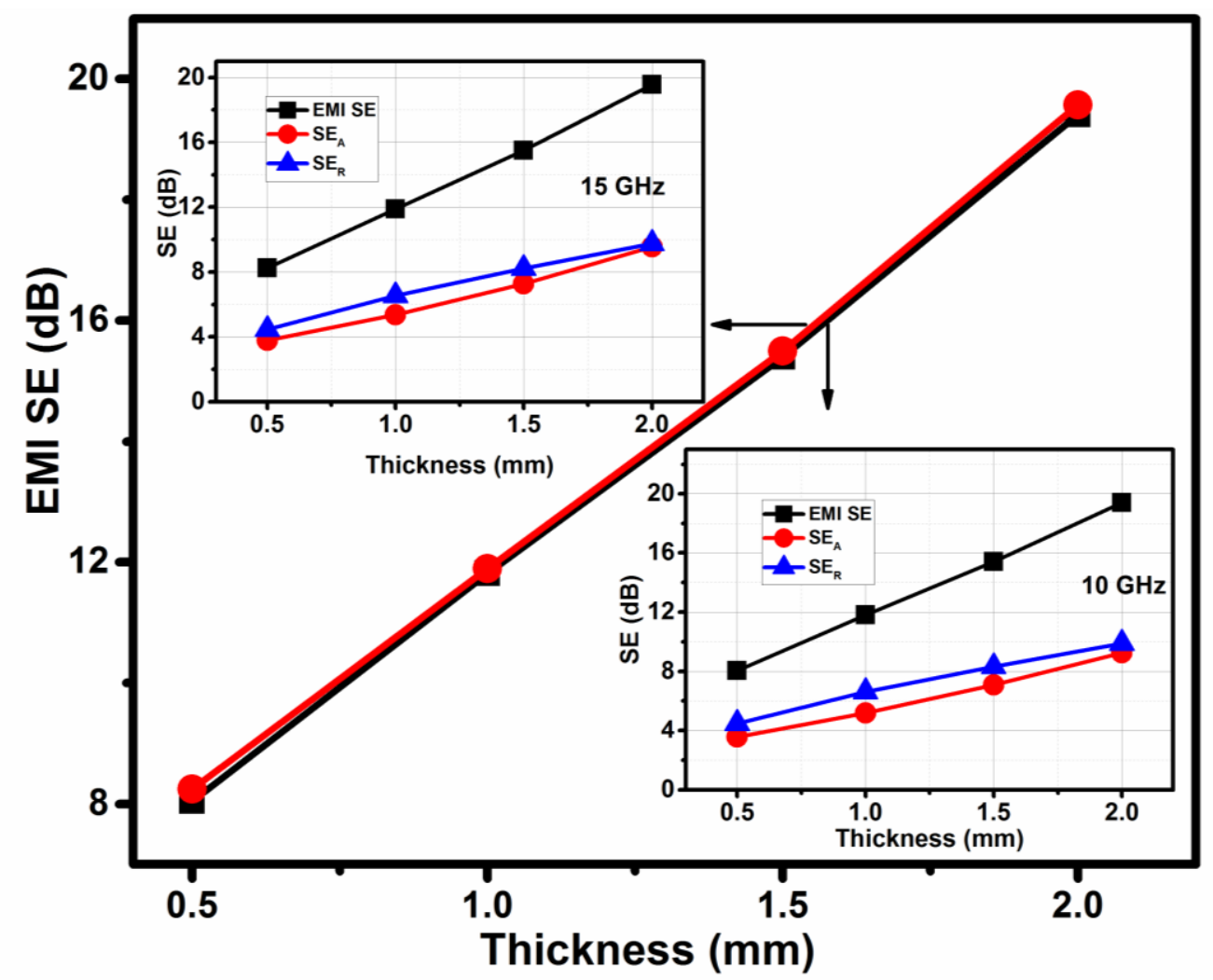

Figure 7: Variation of EMI SE (Inset figures: $E M I, \mathrm{SE}_{\mathrm{A}}$ and $\mathrm{SE}_{\mathrm{R}}$ ) with thickness at two frequencies of 10 and $15 \mathrm{GHz}$ of PVDF-60 SiC composite.

All these results indicate that, the obtained thin paper composites can be used for electrostatic discharge shielding applications and for EMI shielding applications with increased thickness. Hence, PVDF-SIC composite paper films can provide thin and flexible shields by easy and cost effective approach.

\section{Conclusion}

Thin PVDF-SiC shielding composite paper films were prepared by simple solution casting process. The absence of any reaction between the filler and the polymer were confirmed from the XRD and SEM analysis. With increase in filler loading, the composite showed decreasing nature for thermal and mechanical properties and that of increasing trend for $\varepsilon_{\mathrm{r}}, \tan \delta$, conductivity and EMI shielding properties. The PVDF-60 vol\% SiC composite of $1 \mathrm{~mm}$ thickness exhibited DC conductivity of the order of $10^{-5} \mathrm{~S} / \mathrm{m}$ that indicating its potential for electrostatic discharge shielding applications. This was further confirmed from its EMI SE value of $12 \mathrm{~dB}$ corresponding $94 \%$ attenuation. The composite was found to be a partially reflecting and absorbing shielding material. The AC conductivity and skin depth 
value of the composites exhibited an inverse relationship with each other and are major factors effecting the shielding thickness. The increase in sample thickness from 0.5 to $2 \mathrm{~mm}$ resulted in the change of attenuating power of the composite (PVDF-60 SIC) from 84\% to 99\%. Hence, thin and flexible paper like EMI shield of PVDF-SiC composite can be easily prepared by cost effective approach and can be used for various shielding applications like electrostatic discharge shielding as well as EMI shielding.

\section{Reference}

1. Y. Li, H. Yang, X. Hao, N. Sun, J. Du and M. Cao, J. Alloy. Compd. 772, 99 (2019).

2. Y. Mamunyaa, L. Matzuib, L. Vovchenkob, O. Maruzhenkoa, V. Oliynykb, S. Puszc, B. Kumanekc and U. Szelugac, Compos. Sci. Technol. 170, 51 (2019)

3. S. Iqbal, J. Shah, R.K. Kotnala and S.Ahmad, J. Alloy. Compd. 779, 487 (2019).

4. S. Lin, S. Ju, J. Zhang, G. Shi, Y. He and D. Jiang, RSC Adv. 9, 1419 (2019).

5. N. Bagotiaa, V. Choudharyb, D. K. Sharma, Composites Part B, 159, 378 (2019).

6. https://www.et-esd.de/schuhezubehoer/2019

7. R. Ramasubramaniam, J. Chen and H. Liu, Appl. Phys. Lett. 83, 2928 (2003).

8. W. D. Kimmel and D. D. Gerke, Med. Dev. Diag. Ind. 20, 102 (1998)

9. J. E. Vinson and J. J. Liou, Proc IEEE 86, 399 (1998)

10. N. Joseph, J. Chameswary, M. T. Sebastian, Compos. Sci. Technol. 101, 139 (2014).

11. Z. An, C. Ye, R. Zhang and Q. Qu, J. Sol-Gel Sci. Technol. 89, 623 (2019).

12. M. Izawa, T. Koseki, Y. Kamiya and T. Toyomasu. Rev Sci Instrum. 66, 1910 (1995).

13. C. Wang, Y. Liu, Q. You, F. Ye and L. Cheng, Ceram. Int. 45, 5637 (2019)

14. L.Q. Chen, X.W. Yin, X.M. Fan, M. Chen, X.K. Ma, L.F. Cheng and L.T. Zhang, Carbon 95, 10 (2015).

15. H. Wang, D. Zhu, Y. Mu, W. Zhou and F. Luo, Ceram. Int. 41, 14094 (2015).

16. D.H. Ding, Y.M. Shi, Z.H. Wu, W.C. Zhou, F. Luo and J. Chen, Carbon 60, 552 (2013).

17. H. Mei, D.Y. Han, S.S. Xiao, T.M. Ji, J. Tang and L.F. Cheng, Carbon 109, 149 (2016).

18. N. Joseph and M. T. Sebastian, Mater. Lett. 90, 64 (2013).

19. N. Joseph, J. Varghese and M. T. Sebastian, Composites Part B 123, 271 (2017). 
20. N. Joseph, S. K. Singh, R. K. Sirugudu, V. R. K. Murthy, S. Ananthakumar and M. T. Sebastian, Mater. Res. Bull. 48, 1681 (2013).

21. R. K. Goyal, A.N. Tiwari, U.P. Mulik and Y.S. Negi, Comp. Sci. Tech. 67, 1802 (2007).

22. M. Rusu, N. Sofian and D. Rusu, Polym. Test. 20, 409 (2001).

23. K. P. Murali, S. Rajesh, O. Prakash, A. R. Kulkarni and R. Ratheesh, Compos Part A 40, 1179 (2009).

24. M. T Sebastian, Dielectrics for wireless communications; Elsevier: UK, 2008

25. J. Sun, J. Li, G. Sun, B. Zhang, S. Zhang and H. Zhai, Ceram. Int. 28, 741 (2002).

26. N. Joseph, J. Varghese and M.T. Sebastian, J. Mater. Chem. C 4, 999 (2016).

27. N. Joseph, J. Varghese and M.T. Sebastian, Polymer Journal 49, 391 (2017).

28. N. Joseph, J. Varghese and M.T. Sebastian, RSC Adv. 5, 20459 (2015).

\section{Figure captions}

Figure 1: Photographic images of PVDF-SiC composite paper films (a) SiC loading from 10-60 vol\% (b) SiC loading of 10 and $60 \mathrm{vol} \%$ (c-g) indicating its flexibility.

Figure 2: XRD pattern of the PVDF -SiC composite with 60 vol\% filler loading (Inset figures show the SEM fractogram image of the same composite, XRD pattern of pure PVDF and SiC ceramic).

Figure 3: CTE and tensile strength of the of PVDF-SiC composite with different filler loading

Figure 4: Dielectric properties of PVDF-SiC composite with different filler loading in the frequency range of 8.2-18 $\mathrm{GHz}$.

Figure 5: AC conductivity (Inset figure DC conductivity) and skin depth of the PVDF-SiC composite with filler loading in the frequency range of $8-18 \mathrm{GHz}$

Figure 6: EMI SE (Inset figures: $\mathrm{SE}_{\mathrm{A}}$ and $\mathrm{SE}_{\mathrm{A}}$ ) of the PVDF-SiC composite with filler loading at frequencies 8.2, $10,12,15$ and $18 \mathrm{GHz}$.

Figure 7: Variation of EMI SE (Inset figures: EMI, $\mathrm{SE}_{\mathrm{A}}$ and $\mathrm{SE}_{\mathrm{R}}$ ) with thickness at two frequencies of 10 and 15 GHz of PVDF-60 SiC composite. 